\title{
Report of the Third Heart Surgery Forum Scientific Sessions: Zagreb, Croatia December 6-8, 2017: Conference Highlights
}

\author{
Michael S. Firstenberg, MD, FACC, ${ }^{1}$ Tom C. Nguyen, $M D,{ }^{2}$ Harold Roberts, Jr, MD, ${ }^{3}$ \\ Mark M. Levinson, $\mathrm{MD},{ }^{4}$ Igor Rudez, $\mathrm{MD}, \mathrm{PhD}^{5}$ \\ ${ }^{1}$ Department of Surgery, Summa Akron City Hospital, Akron OH; ${ }^{2}$ Department of Cardiothoracic and Vascular Surgery, University \\ of Texas-Memorial Hermann, Houston, TX; ${ }^{3}$ Department of Cardiac Surgery, West Virginia University, Morgantown, WV, USA; \\ ${ }^{4}$ Central Washington Hospital \& Clinics, Wenatchee, WA ${ }^{5}$ Department of Cardiac and Transplant Surgery, University Hospital \\ Dubrava, Zagreb, Croatia
}

\section{ABSTRACT}

The Heart Surgery Forum is an online community dedicated to topics related to all aspects of cardiothoracic surgery. It consists of an informative website (www.hsforum. com), a traditional indexed journal both in print and online, and an email-based "list-serv" for discussion of surgical cases and techniques. The email list-serv, "OpenHeart-L" (The Forum) is composed of surgeons and allied specialties (perfusion, anesthesia, nursing). Dr. Mark Levinson (USA) started The Forum originally in 1995. He also served as the first Editor-in-Chief of the print journal for many years. Coinciding with the popularity of The Forum, and the desire by many members to meet in person, the First Heart Surgery Forum Conference (\#1 HSF) was held in Savudrija, Istria, Croatia in 2010. The overwhelming success of this meeting, in terms of attendance and scientific content, resulted in \#2 HSF being held in Split, Croatia in 2014. Recently, the \#3 HSF meeting was held in Zagreb, Croatia on December 6-8th, 2017. This report highlights the scientific events of this meeting, and more importantly aims to inspire greater involvement by the international cardiothoracic community. Substantial increasing attendance was seen at each subsequent meeting, not only in terms of the number of participants and lectures, but also in the number of countries represented.

\section{INTRODUCTION}

The Third Heart Surgery Forum Conference (\#3 HSF) took place during the brilliant Christmas Market in Zagreb, Croatia. This festival has been ranked the number one Christmas festival in Europe two years in a row. Similar to the two previous meetings that also took place in Croatia, \# 3 HSF featured both international and local faculty who provided updates on the full spectrum of issues that cardiothoracic surgeons face worldwide. Topics ranged from "how-to-do-it" lectures from international experts to open discussions on some of the ethical, economic, and quality

Correspondence: Michael S Firstenberg, MD FACC, Department of Surgery Cardiothoracic, Akron City Hospital-Summa Health System, 75 Arch Street, Suite 407, Akron, Obio 44309; 330-384-9001; fax: 330-384-9002 (email: msfirst@gmail.com). reporting issues that cardiac surgeons face. In addition, local faculty and surgeons-in-training also had opportunities to showcase their work and accomplishments. There were over 180 participants representing all continents (except, of course, Antarctica), many of whom were local medical students and residents (Figure 1). The program was organized and directed by Igor Rudez MD, PhD, of the Department of Cardiac and Transplant Surgery at the University Hospital Dubrava (Croatia). The scientific and organizing committee consisted of his colleagues, Drs. Davor Baric (Croatia), Robert Blazekovic (Croatia), and Daniel Unic (Croatia). Under their leadership, the program was considered by many, including previous meeting attendees, to have been the best HSF meeting yet, with content and open-collegial discussions that rival many other larger society meetings.

The program started with an opening ceremony, in which Dr. Rudez and Dr. Levinson welcomed the faculty, students, industry representatives, and participants to the meeting and recognized the international efforts that have brought the HSF community together to Croatia (Figure 2). The opening lecture was provided by former Dean of the School of Medicine at the University of Zagreb, Professor Davor Milicic, who set the tone for the sessions

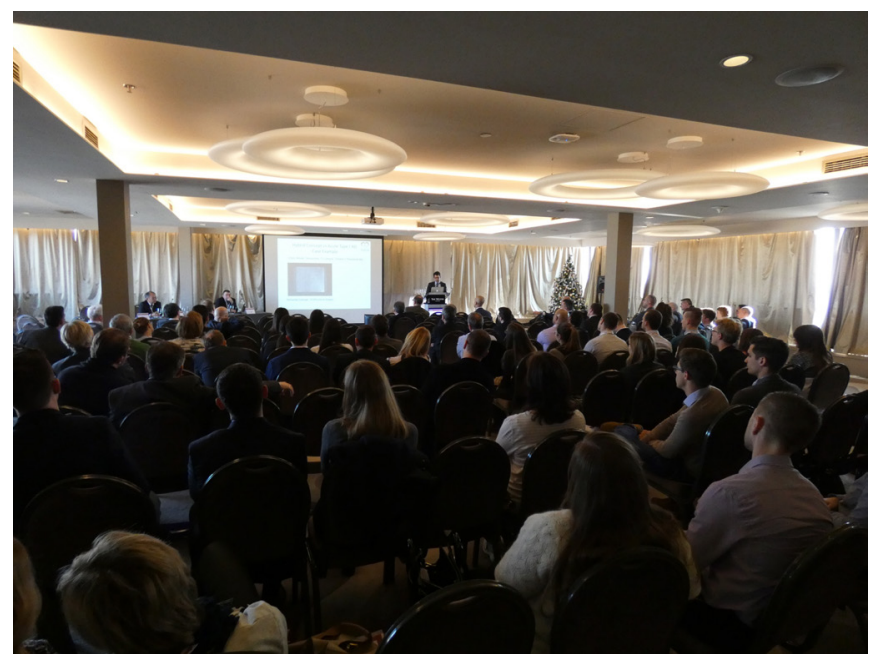

Figure 1. With International representation, there was often standingroom only in the main lecture room. 


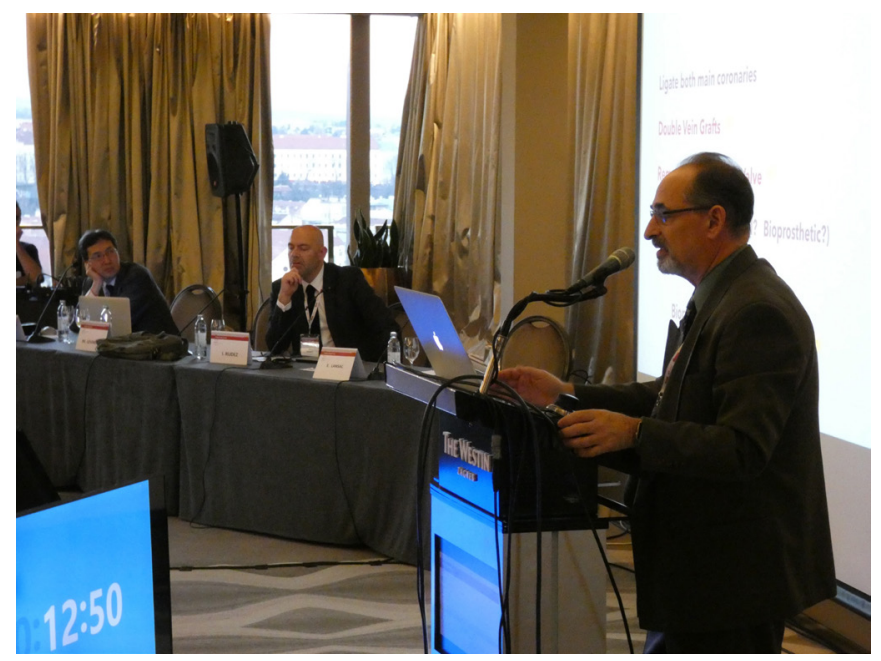

Figure 2. Three Continents of expertise represented in the opening lectures with, right to left, Drs Mark Levinson (at podium) from the United States, Igor Rudez from Croatia, and Tohru Asai from Japan.

with an update that recognized and celebrated 100 years of commitment to excellence at the medical school. This introduction was followed by his Honored Guest Lecture, "Advanced Heart Failure: State of the Art."

Following the introductory lectures that set the tone for the day, the next session focused on aortic valve disease. This session included faculty lectures on evolving techniques and outcomes in sutureless valves, minimally invasive approaches, and some of the clinical challenges that surgeons face, such as treating Jehovah's Witness patients without administering blood transfusions. The session was moderated by Drs. Baric, Simon Moten (Australia), and Enkel Kallushi (Italy) who provided insightful lectures on aortic valve pathology, current guidelines, perspective on rapid deployment valves, as well as discussions on biological versus mechanical valves.

The session on aortic pathology, moderated by Drs. Unic, Marek Ehrlich (Austria), Kay-Hyun Park (Korea), and Konstantinos Tsagakis (Germany) provided extensive insights to the clinical challenges in dealing with both acute and chronic aortic pathologies. They placed particular emphasis on difficult topics ranging from malperfusion syndromes, to the difficulties in operating on Marfan's patients and the long-term problems their difficult condition presents. These presentations emphasized that there is still a tremendous amount that needs to be learned regarding these difficult problems, but the brilliant minds of young students and surgeons continue to take strides toward finding these answers and understanding. Topics covered included acute Type A dissections, surgery for chronic dissections, proper ways to treat malperfusion syndrome, and more.

Dr. Levinson led the next session, focusing on aortic root pathology. He gave an interesting and innovative lecture on the possibility of classifying root lesions in acute aortic dissection. This was followed by Dr. Emmanuel Lansac's (France) lecture, with a plea to standardize aortic valve repair and the role of the AVIATOR registry. Finally, Dr. Rudez discussed the logistics of starting an aortic valve repair program.

The next session focused on some of the technical aspects of mitral and tricuspid valve disease. Dr. Bojan
Biocina (Croatia) presented mid-term results in cross-chordopexy technique. Dr. Tom C. Nguyen (USA) shared pearls and pitfalls from his experience in performing minimally invasive concomitant mitral and tricuspid valve repair. Dr. Tohru Asai (Japan) explained his famous "butterfly-technique," or in other words, how to "resect with respect!" The extremely controversial topic of post-operative management of patients after Cox-Maze cryo-ablations was provided by Dr. Harold Roberts (USA), an internationally recognized expert in the field of arrhythmia surgery. He presented data from a press publication in The Fournal of Thoracic and Cardiovascular Surgery that demonstrated, with a well-managed left atrial appendage, post-op anticoagulation after a CryoMaze or Cox-Maze IV procedure was unnecessary in most patients. From his experience at the West Virginia University Heart and Vascular Institute experience, only patients with mechanical valves, low ejection fraction, or a hypercoagulable state needed anticoagulation after the Maze procedure.

The growing challenges of mechanical circulatory support, particularly left ventricular assist devices and extracorporeal membrane oxygenation, were discussed in a lively session moderated by Drs. Michael Firstenberg (USA), Zeljko Sutlic (Croatia), Marko Kutlesa (Croatia), and Hrvoje Gasparovic (Croatia). The topics discussed ranged from the growing role and potential opportunities for cardiac surgeon involvement in promoting the use of and management of patients who might benefit from short-term support therapies, such as ECMO and other ventricular assist devices.

Faculty lectures illustrated the growing opportunities from improvements in clinical outcomes in patients requiring ECMO. The lectures also touched upon the establishment of American and European guidelines that support a role for such therapies in the setting of acute cardiogenic shock, and for high-risk hybrid and catheter-based interventions. Evidence to support potential under-utilization of support therapies, combined with the unique training and experiences in dealing with extracorporeal perfusion therapies and perfusionists, highlighted the opportunities for surgeons to get more involved in the management of patients outside of the cardiac surgery operating rooms and ICUs, and into the catheterization lab as partners with our interventional cardiology colleagues.

A focused lecture on the topic of the economics of ECMO provided valuable insights into the practical considerations of ECMO, specifically addressing resource utilization and financial issues. Cost/benefit and cost/ quality-adjusted life year (QALY) data, including the analysis from the CESEAR trial, was provided to demonstrate the cost effectiveness of ECMO in comparison to other medical and surgical interventions. Other related topics, including the financial implications to individual hospitals, ECMO programs, and surgeons was presented, providing an opportunity for lively discussion. Core stateof-the-art topics that addressed the development of heart transplant and ECMO programs in the context of challenging political and geographic climates were presented. Additional topics included the next frontier of LVAD therapies, specifically: a better understanding of and opportunities for LVAD weaning; the under-appreciated and perplexing issue of infectious complications of heart transplants in patients who require renal replacement therapies; and the role of hematologic disorders, such as 


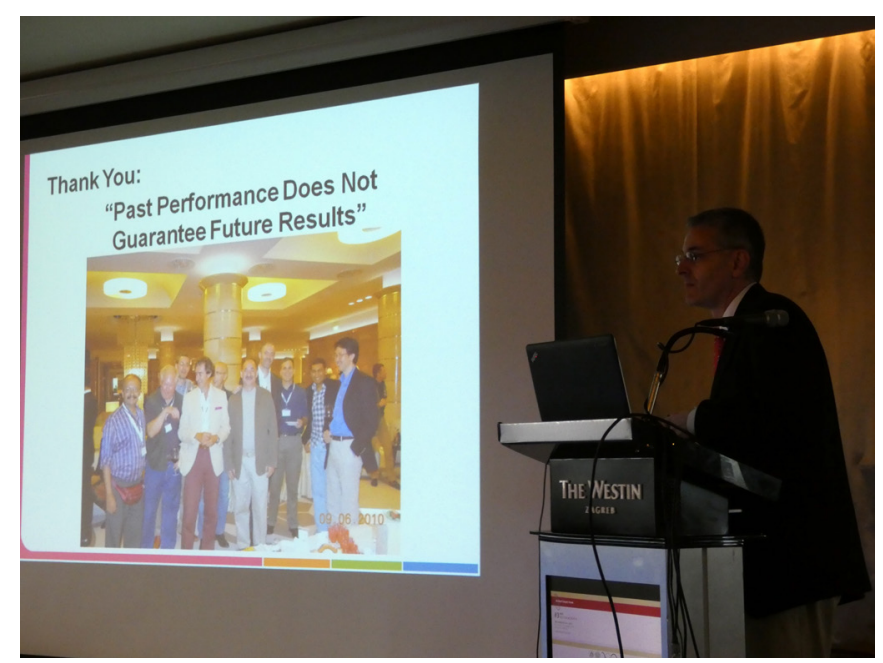

Figure 3. A tribute to the first Heart Surgery Forum meeting in 2010 demonstrated the durability of the personal and professional relationships, as well as the commitment to the organization, as many of the participants at the first meeting, limited only by visa issues, have been able to attend the second and third meetings.

acquired von Willebrand deficiency, in patients requiring cardiothoracic surgical therapies (Figure 3).

One of the most anticipated and attended sessions, affectionately entitled "Tea's Philosophy Corner," was in recognition of the existential and often esoteric insights of Dr. Tea Acuff(USA). Dr. Acuff, a longstanding member and active participant of The Heart Surgery Forum, known for his thoughts on the cognitive aspects of the application of evidence-based literature, presented an overview, "Authority, Logic, Statistics, Artificial Intelligence: Where Should Medicine Find Future." This engaging lecture provided a foundation for lectures by Dr. Ajit Damle (USA), on some of the most important topics that cardiac surgeons are now facing, specifically, "Physician Burnout in the U.S." and "Big Brother Is Watching You! Cardiac Surgeons' Quality Monitoring in the US." Other lectures, including those by Dr. Giuseppe Rescigno (UK), highlighted the challenges that surgeons face when working in different, potentially foreign, environments.



Figure 4. Dr. Rudez presents Dr. Aleksandra Ljubacev with her award for the best overall presentation at the Young Investigator's session.

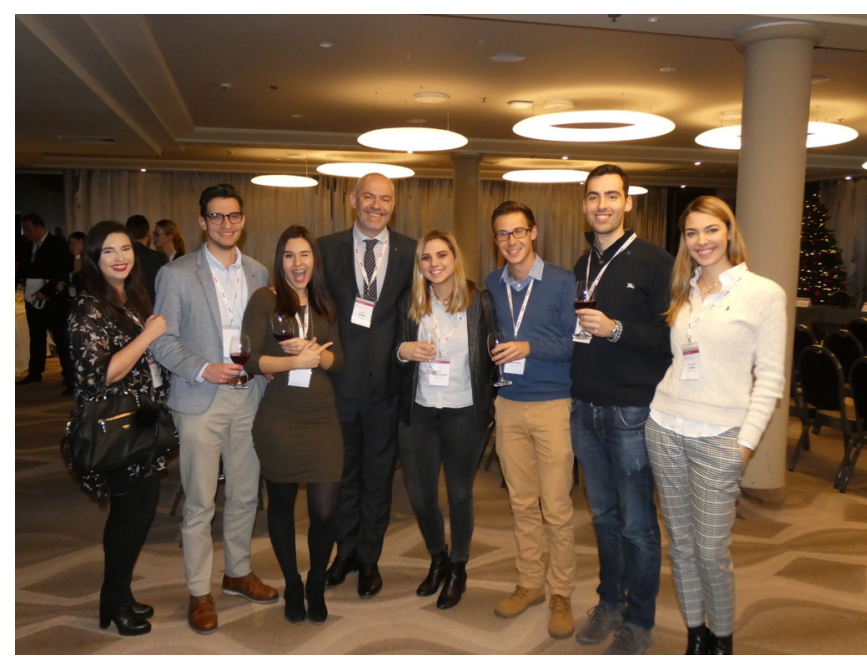

Figure 5. There were numerous opportunities for social interactions among the participants to engage in serious (as well as not so serious) discussions that plant the seeds for life-long professional relationships..

\section{THE YOUNG INVESTIGATORS' SESSION}

The final session, considered by many to have been the most intellectually insightful, was the Young Investigators' Award presentations. Young investigators from local centers, Europe, and South America presented their work on topics that ranged from antiplatelet therapy and testing, neurologic complications after coronary artery bypass surgery, to a full spectrum of case reports and patient series emphasizing the critical importance of cardiac surgeons being both expert technicians and, more importantly, well-rounded clinical doctors and investigators. Dr. Aleksandra Ljubacev (Croatia) gave a particularly insightful presentation, "Comparison of Aortic Valve Repair David Surgery and Biological Bentall Procedure with Echocardiography Follow-up After 6 Year Practice at Out Center." The collection of excellent presentations by some of the upcoming leaders clearly illustrates the promising future of cardiothoracic surgery (Figure 4).

While the scientific content was considered by some to rival, if not exceed, the quality and depth typically encountered at much larger society meetings, \#3 HSF was not all work and no play. A core foundation of The Forum is bringing surgical colleagues, young and old, from all over the world, to build personal and professional relationships. It is not unusual for members to visit their friends' and colleagues' programs and homes on the other side of the world. Recognizing that the future of cardiac surgery rests in the youth and their open minds and enthusiasm, residents and fellows are welcomed with open arms (Figure 5). To encourage such interactions and relationship development, several social programs were organized throughout the entire meeting to coincide with the Christmas holiday spirit that Zagreb had to offer. To mention a few highlights of these social activities, these young residents and fellows shared meals at the the Zagreb Westin's (the conference venue) restaurant, ventured on a group excursion to the Tesla Museum, and celebrated at several dinner parties. All conference attendees, regardless of their age, were encouraged to attend these social events. Without a doubt, the meeting was a phenomenal opportunity to make new friends, reunite with old friends, and bond with colleagues from around the world (Figure 6). 


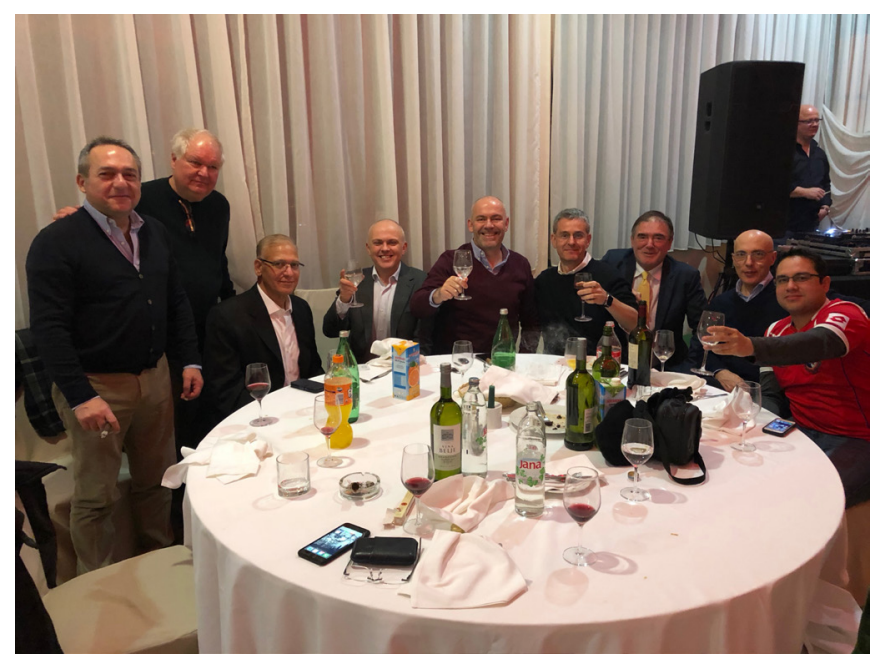

Figure 6. Friends and colleagues from across town and from across the globe gather to celebrate the season and the success of the Third Heart Surgery Forum meeting.

\section{PUBLICATIONS}

All accepted abstracts were published in Volume 20, Issue 5 of The Heart Surgery Forum (HSF), which is available online at http://www.hsforum.com. All present and past issues of the HSF are indexed by several search engines, including Index Medicus (Pub Med), Medline, Embase, SciSearch, and ISI. Authors are encouraged to submit full manuscripts for peer review at the HSF home page. As of January 2017, the HSF is now open access, allowing readers full access to the latest science in cardiothoracic surgery. Full manuscripts can be viewed without a subscription fee.

\section{CONCLUSIONS}

Despite the challenges of traveling to Croatia in the winter season, those who traveled from all over the globe were rewarded with a scientific conference unmatched in terms of the collegiality and opportunity to build, maintain, and reinvigorate lasting personal and professional relationships. The Heart Surgery Forum is one of the first online, digital, scientific social media communities. It is dedicated to the goal of bringing together surgeons, at all levels, from all over the world, to share common interests and topics of discussion in a manner that is open, supportive, educational, provocative, and collegial. While at times, the online discussions can be confrontational and challenging of beliefs, members come together as support friends online on a daily basis. The opportunity to get together in person is a natural extension of these online interactions. Many Forum members have met in person and supported one another, both personally and professional, all over the world. The \#3 HSF Conference in Zagreb was an opportunity to bring everyone together in a common and beautiful location. Discussions are already underway for the Heart Surgery Forum Conference and while it will be difficult to improve on the \#3 HSF - many are up to the challenge and all are encouraged to get involved. 